\title{
Cosmological perturbations in brane-world theories: Formalism
}

\author{
Carsten van de Bruck, ${ }^{1,2, *}$ Miquel Dorca, ${ }^{1, \dagger}$ Robert H. Brandenberger, ${ }^{1, \sharp}$ \\ and André Lukas ${ }^{3, \S}$ \\ ${ }^{1}$ Department of Physics, Brown University, Providence, Rhode Island 02912 \\ ${ }^{2}$ DAMTP, Center for Mathematical Sciences, Wilberforce Road, CB3 OWA, Cambridge, United Kingdom \\ ${ }^{3}$ Department of Physics, Theoretical Physics, University of Oxford 1 Keble Road, Oxford OX1 3NP, United Kingdom
}

(Received 19 June 2000; published 29 November 2000)

\begin{abstract}
We develop a gauge-invariant formalism to describe metric perturbations in five-dimensional brane-world theories. In particular, this formalism applies to models originating from heterotic $\mathrm{M}$ theory. We introduce a generalized longitudinal gauge for scalar perturbations. In this gauge, we derive the five-dimensional evolution equations for scalar perturbations as well as the most general structure of the scalar brane stress-energy tensor. As an application, we discuss some aspects of the evolution of fluctuations on the brane. Moreover, we show how the five-dimensional formalism can be matched to the known four-dimensional one in the limit where an effective four-dimensional description is appropriate.
\end{abstract}

PACS number(s): 98.80.Cq, 98.65.-r

\section{INTRODUCTION}

In recent years, the way in which string theory is believed to be connected to observable physics has changed dramatically. The new viewpoint is mainly due to two ideas: namely the brane-world idea [1-7] and the idea that couplings and scales of additional dimensions are much more flexible than previously assumed $[2,8,5,6]$. Not only have these ideas led to new directions in M-theory phenomenology and, more generally, string-theory inspired particle phenomenology, but also in early universe cosmology.

Much of the recent activity in brane-world cosmology is centered around five-dimensional brane-world theories, see for example [9-37]. A large class of such theories arises from heterotic $\mathrm{M}$ theory $[4,38,39]$. Other five-dimensional models have been introduced in Refs. [40-43] which may provide an alternative solution to the hierarchy problem.

A central question is whether the possible existence of a brane world and large additional dimensions in the early universe leads to observable consequences today. Specifically, cosmological perturbations as, for example, observed in the cosmic microwave background provide us with a window to the early universe that, perhaps, can be used to test the braneworld idea. It is with this motivation in mind that we set out to study metric perturbations in brane-world models. It may not be immediately clear that the existence of additional dimensions and branes should have important consequences for the formation and evolution of cosmological perturbations. Let us, as a comparison, consider "traditional" Kaluza-Klein cosmologies, where the higher-dimensional universe is usually split into a product of maximally symmetric subspaces each one with an individual scale factor. Cosmological perturbations are normally treated at the linearized level and, hence, in such Kaluza-Klein cosmologies [47-49]

\footnotetext{
*Email address: C.VanDeBruck@damtp.cam.ac.uk

†Email address: dorca@het.brown.edu

‡Email address: rhb@het.brown.edu

${ }^{\S}$ Email address: lukas@thphys.ox.ac.uk
}

internal and external fluctuations basically decouple. The additional dimensions effect perturbations of the threedimensional universe only via the kinematics of the background scale factors (and/or dilaton background fields [50]). The situation is quite different for brane-world theories. The branes are stretched across the three-dimensional universe and are located at specific points in the internal space. Moreover, they carry world volume fields that can only propagate on the brane and that are likely to be excited in the early universe, both coherently and thermally. As a consequence, the cosmological background in an early brane-world universe is highly inhomogeneous in the additional dimensions since the branes constitute localized sources of stress-energy. Even if perturbations around such a background are treated at the linearized level, the perturbations of the threedimensional universe are effected by the non-linear distortion of the cosmological background in the internal dimensions. This constitutes a crucial difference between conventional Kaluza-Klein cosmology and brane-world cosmology which is directly related to the presence of branes. It is this difference that may lead to new predictions for cosmological perturbations in brane-world models and that motivates the present investigation.

In this paper, we will develop a formalism for metric fluctuations in brane-world theories that takes the characteristic property of brane-world cosmologies, the above mentioned inhomogeneity in the additional dimensions, into account. We understand such a formal development as a first step towards analyzing predictions for cosmological perturbations in brane-world theories. In the next section, we start out to generalize the well-known formalism of fourdimensional gauge-invariant metric perturbations $[44,45]$ to brane-world theories with an arbitrary number of additional dimensions. Subsequently, in Sec. III, we focus on fivedimensional brane-world models on the orbifold $S^{1} / Z_{2}$ related to those originating from heterotic $M$ theory $[4,38,39]$. Specifically, we consider the five-dimensional Einstein equation coupled to bulk as well as brane stress-energy. For such a theory we derive the equations of motion for scalar metric perturbations in a generalized longitudinal gauge. Consis- 
tency of these equations is used to determine the most general structure of the stress-energy on the brane. In Sec. IV, these results are applied to find the Israel matching conditions [46] for the scalar metric perturbations restricted to the branes. Finally, in Sec. V, we show how our formalism for brane-world metric perturbations is related to the conventional one in four dimensions. This is done by a matching procedure applied in a limit where the five-dimensional brane-world theory has an effective four-dimensional description.

\section{GAUGE-INVARIANT VARIABLES}

In this section, we will develop a gauge-invariant formalism for metric perturbations in brane-world models. Using such a gauge-invariant approach is particularly useful in order to identify the correct physical degrees of freedom. Once this has been done, a specific gauge can be chosen in order to simplify the subsequent equations. Specifically, we will use a generalized longitudinal gauge later on. However, as a warm-up for the higher-dimensional case, we would first like to review the well-known four-dimensional gauge-invariant formalism following Ref. [45].

\section{A. The four-dimensional formalism}

Starting point is a background metric with a maximally symmetric three-dimensional spatial space. This metric is of the form

$$
d s_{4}^{2}=a_{4}^{2}\left\{d t^{2}-\Omega_{i j} d x^{i} d x^{j}\right\}
$$

where indices $i, j, \ldots=1,2,3$ run over the three spatial indices. Indices $\mu, \nu, \ldots=0,1,2,3$ are used to index fourdimensional space-time. Furthermore, $a_{4}=a_{4}(t)$ is the fourdimensional scale factor and $\Omega_{i j}$ is the metric of the threedimensional maximally symmetric space explicitly given by

$$
\Omega_{i j}=\delta_{i j}\left[1+\frac{1}{4} k x^{l} x^{m} \delta_{l m}\right]^{-2} .
$$

Here $k=0,1,-1$ corresponds to a flat, closed or open universe, respectively. The idea is now to classify perturbations of the metric (1) according to their transformation properties with respect to the maximally symmetric space. This leads to the perturbed metric

$$
\begin{aligned}
d s_{4}^{2}= & a_{4}^{2}\left\{\left(1+2 \phi_{4}\right) d t^{2}-\left[\left(1-2 \psi_{4}\right) \Omega_{i j}+2 E_{4 \mid i j}+2 F_{4(i \mid j)}\right.\right. \\
& \left.\left.+h_{4 i j}\right] d x^{i} d x^{j}+W_{4 i} d t d x^{i}\right\} .
\end{aligned}
$$

Here and in the following four-dimensional quantities are indexed by " 4 " to distinguish them from their higherdimensional counterparts to be introduced later. The vertical bar refers to a covariant derivative with respect to the metric $\Omega_{i j}$. The vector $F_{4 i}$ has a vanishing divergence, that is $F_{4 i}{ }^{i}=0$ and the tensor $h_{4 i j}$ is traceless and divergence-less, that is $h_{4 i}{ }^{i}=0$ and $h_{4 i \mid j}^{j}=0$. In addition, we can decompose the off-diagonal perturbation $W_{4 i}$ further into the gradient of a scalar $B_{4}$ and a divergence-less vector $S_{4 i}$. Explicitly, this reads

$$
W_{4 i}=B_{4 \mid i}+S_{4 i} \text {. }
$$

Consequently, we have four scalar metric perturbations $\left(\phi_{4}, \psi_{4}, E_{4}, B_{4}\right)$, two vector perturbations $\left(F_{4 i}, S_{4 i}\right)$ and a tensor perturbation $\left(h_{4 i j}\right)$. All these perturbations are functions of time as well as of the spatial coordinates $x^{i}$, of course. Next we consider an infinitesimal coordinate transformation

$$
x^{\mu} \rightarrow \tilde{x}^{\mu}=x^{\mu}+\xi^{\mu},
$$

where the vector $\xi^{\mu}$ depends on all four coordinates, in general. The corresponding infinitesimal change of the metric is given by

$$
g_{4 \mu \nu} \rightarrow \tilde{g}_{4 \mu \nu}=g_{4 \mu \nu}-2 \nabla_{(\mu} \xi_{\nu)} .
$$

To understand how this coordinate transformation acts on the metric perturbations we split $\xi^{\mu}$ as $\xi^{\mu}=\left(\xi^{0}, \xi^{i}\right)$ into a time and a spatial part. The spatial component $\xi^{i}$ can be decomposed further into a gradient and a divergence-less part as

$$
\xi^{i}=\xi^{i}+\eta^{i}
$$

As a result, the transformation parameter $\xi^{\mu}$ contains two scalar components $\left(\xi^{0}, \xi\right)$ and one vector component $\left(\xi^{i}\right)$. Given this setup, one can compute the transformation properties of the metric perturbations by applying Eq. (6) to the perturbed metric (3) and taking into account that $\xi_{\mu}$ $=a_{4}^{2}\left(\xi_{0},-\xi_{i}\right)$. For the scalar perturbations one finds

$$
\begin{aligned}
& \widetilde{\phi}_{4}=\phi-H_{4} \xi^{0}-\xi^{0}, \\
& \widetilde{\psi}_{4}=\psi_{4}+H_{4} \xi^{0}, \\
& \widetilde{B}_{4}=B_{4}+\xi^{0}-\dot{\xi}, \\
& \widetilde{E}_{4}=E_{4}-\xi .
\end{aligned}
$$

Here, $H_{4}$ is the Hubble parameter defined by $H_{4}=\dot{a}_{4} / a_{4}$. The vector perturbations transform as

$$
\begin{gathered}
\widetilde{F}_{4 i}=F_{4 i}-\eta_{i}, \\
\widetilde{S}_{4 i}=S_{4 i}-\dot{\eta}_{i},
\end{gathered}
$$

while the tensor perturbation $h_{4 i j}$ is invariant. In these equations, spatial indices are lowered and raised with the metric $\Omega_{i j}$, that is, for example $\xi_{\mid i}=\Omega_{i j} \xi^{\mid j}$ and $\eta_{i}=\Omega_{i j} \eta^{j}$. With these results, it is straightforward to introduce the following gauge-invariant variables.

\section{Scalar variables}

$$
\begin{aligned}
& \Phi_{4}=\phi_{4}+H_{4}\left(B_{4}-\dot{E}_{4}\right)+\dot{B}_{4}-\ddot{E}_{4} \\
& \Psi_{4}=\psi_{4}-H_{4}\left(B_{4}-\dot{E}_{4}\right) .
\end{aligned}
$$

Vector variables 


$$
\mathcal{F}_{i}=S_{4 i}-\dot{F}_{4 i}
$$

Tensor variables

$$
h_{4 i j} \text {. }
$$

As the physical degrees of freedom, one has therefore identified two scalar perturbations, one vector perturbation and one tensor perturbation. Of particular importance are the two scalar perturbations $\Phi_{4}$ and $\Psi_{4}$ on which we are going to focus. The expressions (14) and (15) for these perturbations suggest the gauge choice $B_{4}=E_{4}=0$ in the scalar sector which is referred to as longitudinal gauge. Clearly, from the above transformation properties of the scalar perturbations such a choice can be made. Then, the gauge-invariant scalar variables coincide with the "original" variables, that is, $\Phi_{4}=\phi_{4}$ and $\Psi_{4}=\psi_{4}$. This gauge choice considerably simplifies subsequent calculations and its generalization will be quite helpful to deal with the higher-dimensional case. The perturbed metric then takes the form

$$
d s_{4}^{2}=a_{4}^{2}\left\{\left(1+2 \phi_{4}\right) d t^{2}-\left(1-2 \psi_{4}\right) \Omega_{i j} d x^{i} d x^{j}\right\} .
$$

Finally, we need to specify the stress-energy. For the background, by the maximal symmetry of the three-dimensional spatial space, it is dictated to be of the form

$$
T_{4}{ }_{\nu}{ }_{\nu}=\operatorname{diag}\left(\rho_{4},-p_{4},-p_{4},-p_{4}\right) .
$$

The scalar perturbations to this stress-energy can be written as

$$
\delta T_{4}{ }^{\mu}{ }_{\nu}=\left(\begin{array}{cc}
\delta \rho_{4} & -\left(\rho_{4}+p_{4}\right) a_{4}^{-1} v_{4 \mid j} \\
\left(\rho_{4}+p_{4}\right) a_{4} v_{4}{ }^{\mid i} & -\delta p_{4} \delta^{i}{ }_{j}+\sigma_{4}{ }^{\mid i} \mid j
\end{array}\right)
$$

with the potential $v_{4}$ for the velocity field $v_{4 \mid i}$ and the quantity $\sigma$ specifying the anisotropic stress. The equations of motion for the background and the scalar perturbations subject to the above stress-energy are given in Ref. [45] and will not be repeated here. These equations form the basis for the study of cosmological perturbations and we now turn to develop their higher-dimensional generalization.

\section{B. Gauge-invariant variables in brane-world theories}

We would now like to proceed in close analogy with the four-dimensional case reviewed above and develop a gaugeinvariant formalism of metric perturbations in brane-world theories. First, we consider the general situation of $d$ additional dimensions although later we will be more specific and focus on the case $d=1$, that is, a five-dimensional universe. The coordinates of the additional dimensions are denoted by $\left(y^{5}, \cdots, y^{4+d}\right)$. For the purpose of this subsection, all we need to specify is that the branes are stretched across the usual four-dimensional space-time and are located at specific points (or submanifolds) in the additional dimensions. We will be more precise about the brane positions later when we consider the five-dimensional case.

How should metric perturbations be classified in such a brane-world theory? In the previous four-dimensional case we have used their tensor properties with respect to the threedimensional spatial subspace for this classification. At first glance, one might now want to use their tensor properties with respect to the $(3+d)$-dimensional spatial space. The cosmological principle, of course, only asserts the maximal symmetry of the usual three-dimensional space but the maximal symmetry of the $d$-dimensional internal space may be taken as an additional, simplifying assumption. It is at this point, that the brane-world nature of the theory comes into the game. Since the branes are localized in the additional dimensions the assumption of maximal symmetry cannot, in general, be extended to those dimensions. In fact, as will become more explicit below, the branes lead to stress-energy in the Einstein equation localized in the additional dimensions and, hence, the symmetry of the background metric will typically not be enhanced with respect to the fourdimensional case. Consequently, we split the coordinates into two groups, namely the inhomogeneous coordinates $\left(y^{a}\right)=\left(t, y^{5}, \cdots, y^{4+d}\right)$ on which the background metric generally depends in a non-trivial way and the usual three spatial coordinates $\left(x^{i}\right)$ corresponding to the maximally symmetric space. In the following we use indices $a, b, \ldots=0,5, \ldots, 4$ $+d$ for time and the additional dimensions, indices $i, j, \ldots$. $=1,2,3$ for the three-dimensional space and indices $\alpha, \beta, \ldots=0,1,2,3,5, \ldots, 4+d$ for the full $(4+d)$ dimensional space-time. Then the most general higherdimensional metric consistent with the maximally symmetric three-dimensional spatial manifold is given by

$$
d s^{2}=a^{2}\left\{\gamma_{a b} d y^{a} d y^{b}-\Omega_{i j} d x^{i} d x^{j}\right\},
$$

where the scale factor $a$ and the metric $\gamma_{a b}$ are functions of the coordinates $y^{a}$ only. Here $\Omega_{i j}$ is the metric of the maximally symmetric space of constant curvature given in Eq. (2). Given this structure of the background metric, we are forced to classify metric perturbations by their threedimensional tensor properties as in the four-dimensional case. We stress again that this is a direct consequence of the brane-world nature of the theory that we are considering. With these remarks in mind, the higher-dimensional generalization of the perturbed metric (3) can be written in the form

$$
\begin{aligned}
d s^{2}= & a^{2}\left\{\gamma_{a c}\left(\delta_{b}^{c}+2 \phi_{b}^{c}\right) d y^{a} d y^{b}-\left[(1-2 \psi) \Omega_{i j}+2 E_{\mid i j}\right.\right. \\
& \left.\left.+2 F_{(i \mid j)}+h_{i j}\right] d x^{i} d x^{j}-2 W_{a i} d y^{a} d x^{i}\right\} .
\end{aligned}
$$

As in the four-dimensional case, $F_{i}$ and $h_{i j}$ have a vanishing divergence and, in addition, $h_{i j}$ is traceless. As before, the three-vectors $W_{a i}$ can be split as follows:

$$
W_{a i}=B_{a \mid i}+S_{a i},
$$

where $S_{a \mid i}{ }_{i}=0$. Observe that the perturbed metric (22), defined in this way, is completely general. In fact, this can be easily seen by counting degrees of freedom. As an example, we can consider the simplest case of only one extra dimension setting $y=y^{5}$ and $a, b, \ldots=0,5$. Then, the most general perturbed metric contains 15 degrees of freedom, which are parameterized by the seven scalar perturbations $\left(\phi_{0}^{0}, \phi_{5}^{0}\right.$, 
$\left.\phi_{5}^{5}, \psi, E, B_{a}\right)$, six degrees of freedom from the vector perturbations $\left(F_{i}, S_{a i}\right)$ and two degrees of freedom from the tensor perturbation $h_{i j}$. Of course, in counting the degrees of freedom originating from vector and tensor perturbations we have taken the constraints on these quantities into account.

Let us now return to the general case of $d$ additional dimensions and consider the coordinate transformations

$$
x^{\alpha} \rightarrow x^{\tilde{\alpha}}=x^{\alpha}+\xi^{\alpha}
$$

with infinitesimal parameters $\xi^{\alpha}$. In accordance with the above discussion, we split these parameters as $\left(\xi^{\alpha}\right)$ $=\left(\xi^{a}, \xi^{i}\right)$. We adopt the useful convention that indices of type $a(i)$ are lowered, raised and contracted using the metric $\gamma_{a b}\left(\Omega_{i j}\right)$. Furthermore, we take the vertical bar to denote the covariant derivative with respect to $\gamma_{a b}$ or $\Omega_{i j}$ depending on the index type. From the transformation law

$$
\tilde{g}_{\alpha \beta}=g_{\alpha \beta}-2 \nabla_{(\alpha} \xi_{\beta)},
$$

of the metric, and taking into account that $\xi_{\alpha}=a^{2}\left(\xi_{a}\right.$, $-\xi_{i}$ ), we find for the transformation of the scalar perturbations

$$
\begin{aligned}
\delta \phi_{a b} & =-\xi_{(a \mid b)}-H^{c} \xi_{c} \gamma_{a b}, \\
\delta \psi & =H^{a} \xi_{a}, \\
\delta E & =-\xi \\
\delta B_{a} & =\xi_{a}-\xi_{\mid a},
\end{aligned}
$$

where we have introduced the generalized Hubble parameters

$$
H_{c}=\frac{a_{\mid c}}{a} .
$$

The vector perturbations in the metric (22) change according to

$$
\begin{gathered}
\delta F_{i}=-\eta_{i}, \\
\delta S_{a i}=-\eta_{\left.i\right|_{a}} .
\end{gathered}
$$

Finally, the tensor perturbation $h_{i j}$ is invariant under the first order gauge transformation (25). From these results we easily find the following gauge-invariant quantities.

\section{Scalar variables}

$$
\begin{aligned}
\Phi_{a b} & =\phi_{a b}+H^{c}\left(B_{c}-E_{\mid c}\right) \gamma_{a b}+\left(B_{(a}-E_{\mid(a}\right)_{\mid b)} \\
\Psi & =\psi-H^{c}\left(B_{c}-E_{\mid c}\right) .
\end{aligned}
$$

Vector variables

$$
\mathcal{F}_{a i}=S_{a i}-F_{i \mid a}
$$

Tensor variables

$$
h_{i j}
$$

We conclude that the physical degrees of freedom consist of the $(d+1)(d+2) / 2+1$ gauge invariant scalar perturbations $\left(\Phi_{a b}, \Psi\right), d+1$ gauge invariant vector perturbations $\mathcal{F}_{a i}$ and a gauge invariant tensor perturbation $h_{i j}$. The above gaugeinvariant variables are a direct generalization of the corresponding four-dimensional ones. Specifically, restricting to no additional dimensions and setting $\gamma_{00}=1$, Eqs. (33) $-(36)$ exactly reproduce the four-dimensional expressions (14)(17). However, in the case $d>0$ our formalism clearly has a richer structure than the conventional four-dimensional one.

\section{A generalized longitudinal gauge for scalar perturbations}

In the subsequent sections we will focus on the evolution of scalar perturbations. Vector and tensor perturbations will be discussed elsewhere. In order to simplify this discussion we introduce a generalized longitudinal gauge for the scalar perturbations. In analogy with the four-dimensional case, this gauge is specified by

$$
B_{a}=0, \quad E=0 .
$$

Setting these quantities to zero can indeed be achieved by an appropriate choice of the scalar transformation parameters $\xi_{a}$ and $\xi$ in Eqs. (26)-(29). Note that we have exactly the correct number of transformation parameters to do this and that, consequently, the gauge ambiguity in the scalar sector is complete eliminated by this choice. Then, the scalar part of the metric takes the simple form

$$
d s^{2}=a^{2}\left\{\gamma_{a c}\left(\delta_{b}^{c}+2 \phi_{b}^{c}\right) d y^{a} d y^{b}-(1-2 \psi) \Omega_{i j} d x^{i} d x^{j}\right\} .
$$

Furthermore, in this gauge, the scalar perturbations $\phi_{a b}$ and $\psi$ coincide with their gauge-invariant counterparts, that is

$$
\Phi_{a b}=\phi_{a b}, \quad \Psi=\psi
$$

as it is the case in four dimensions.

\section{The five-dimensional case}

Let us restrict in this section and for the rest of the paper to the case of a single extra dimension $y=y^{5}$. Then, the indices $a, b, \ldots$ run over the values 0,5 only. Furthermore, in order to be more explicit, we choose the conformal gauge

$$
\left(\gamma_{a b}\right)=b^{2} \operatorname{diag}(1,-1)
$$

for the background metric $\gamma_{a b}$ by performing a large gauge transformation. Here $b=b(t, y)$ is a new, independent scale factor. Then, the perturbed five-dimensional metric (22) reduces to

$$
\begin{aligned}
d s^{2}= & a^{2}\left\{b^{2}\left[(1+2 \phi) d t^{2}-2 W d t d y-(1-2 \Gamma) d y^{2}\right]\right. \\
& -\left[\Omega_{i j}(1-2 \psi)+2 E_{\mid i j}+2 F_{(i \mid j)}+h_{i j}\right] d x^{i} d x^{j} \\
& \left.-2 W_{0 i} d t d x^{i}-2 W_{5 i} d y d x^{i}\right\},
\end{aligned}
$$

where we have defined

$$
\phi=\phi_{0}^{0}, \quad \Gamma=-\phi_{5}^{5}, \quad W=2 \phi_{0}^{5}=-2 \phi_{5}^{0} .
$$


Recall that the scale factors $a$ and $b$ are functions of the coordinates $t$ and $y$ only while the perturbations depend on all spacetime coordinates. The scalar gauge-invariant variables defined in Eq. (33) can now be written more explicitly as

$$
\begin{aligned}
\Phi_{1} \equiv & \Phi_{0}^{0} \\
= & \phi+\frac{1}{b^{2}}\left[\left(H_{0}-\mathcal{H}_{0}\right)\left(B_{0}-\dot{E}\right)+\dot{B}_{0}-\ddot{E}\right. \\
& \left.-\left(H_{5}+\mathcal{H}_{5}\right)\left(B_{5}-E^{\prime}\right)\right] \\
\Phi_{2} \equiv & \Phi_{5}^{5} \\
= & \Gamma-\frac{1}{b^{2}}\left[\left(H_{5}-\mathcal{H}_{5}\right)\left(B_{5}-E^{\prime}\right)+B_{5}^{\prime}-E^{\prime \prime}\right. \\
& \left.-\left(H_{0}+\mathcal{H}_{0}\right)\left(B_{0}-\dot{E}\right)\right] \\
\Phi_{3} \equiv & \Phi_{0}^{5} \\
= & \frac{W}{2}-\frac{1}{2 b^{2}}\left[\left(B_{0}^{\prime}+\dot{B}_{5}\right)-2 \dot{E}^{\prime}-2 \mathcal{H}_{5}\left(B_{0}-\dot{E}\right)\right. \\
& \left.-2 \mathcal{H}_{0}\left(B_{5}-E^{\prime}\right)\right] \\
\Phi_{4} \equiv & \Psi=\psi-\frac{1}{b^{2}}\left[H_{0}\left(B_{0}-\dot{E}\right)-H_{5}\left(B_{5}-E^{\prime}\right)\right] .
\end{aligned}
$$

Here and in the following the dot (prime) denotes the derivative with respect to time (the coordinate $y$ ). Furthermore, we have introduced a second set of "Hubble" parameters $\mathcal{H}_{a}$ $=b_{\mid a} / b$. Let us specialize these results to the generalized longitudinal gauge defined by $B_{0}=B_{5}=E=0$. Then the above scalar gauge-invariant variables coincide with $\phi, \psi, \Gamma$ and $W$. The metric simplifies to

$$
\begin{aligned}
d s^{2}= & a^{2}\left\{b^{2}\left[(1+2 \phi) d t^{2}-2 W d y d t-(1-2 \Gamma) d y^{2}\right]\right. \\
& \left.-(1-2 \psi) \Omega_{i j} d x^{i} d x^{j}\right\} .
\end{aligned}
$$

This metric will be the starting point for our treatment of scalar perturbations in the following sections. In addition to the perturbations $\phi$ and $\psi$ that we are familiar with from the four-dimensional case it contains two new perturbations, $\Gamma$ and $W$, that are related to the presence of the fifth dimension.

\section{THE PERTURBED EINSTEIN EQUATION IN THE LONGITUDINAL GAUGE}

As we have previously mentioned, the main application we have in mind for this paper is a compactification of a five-dimensional theory on the orbifold $S_{1} / Z_{2}$. We start by compactifying the fifth dimension on a circle restricting the corresponding coordinate $y$ to the range $y \in[-R, R]$ with the endpoints identified. The action of the $Z_{2}$ orbifolding symmetry on the circle is taken to be $y \rightarrow-y$. Consequently, there exist two fix points at $y=y_{1}=0$ and $y=y_{2}=R$. We assume that the three-branes, stretching across $(3+1)$-dimensional space-time, are located at these fix points in the orbifold direction. This setup is appropriate for a large class of five-dimensional heterotic M-theory models $[4,38,39]$ that originate from 11-dimensional Horava-Witten theory. It also applies to the five-dimensional models introduced in Refs. [41,42].

Next, we should truncate the five-dimensional metric in order to make it consistent with the orbifolding. Since the metric has to be intrinsically even under the $Z_{2}$ action its various components satisfy the constraints

$$
\begin{aligned}
& g_{\mu \nu}(-y)=g_{\mu \nu}(y), \\
& g_{\mu 5}(-y)=-g_{\mu 5}(y), \\
& g_{55}(-y)=g_{55}(y) .
\end{aligned}
$$

At the same time, we have to make sure that coordinate transformations do not lead out of the class of metrics defined this way. The parameter $\xi^{\alpha}$ for an infinitesimal coordinate transformation has, therefore, to be restricted by

$$
\begin{aligned}
& \xi^{\mu}(-y)=\xi^{\mu}(y), \\
& \xi^{5}(-y)=-\xi^{5}(y),
\end{aligned}
$$

which directly follows from Eq. (25). From these rules we can deduce the $Z_{2}$ properties of the various quantities in metric (47) for scalar perturbations. While the background scale factors $a, b$ as well as the perturbations $\phi, \psi$ and $\Gamma$ are $Z_{2}$ even, that is, for example, $a(-y)=a(y)$, the perturbation $W$ is $Z_{2}$ odd, that is $W(-y)=-W(y)$. Similarly, for the scalar components in the transformation parameter $\xi^{\alpha}$, we find that $\xi_{0}$ and $\xi$ are even while $\xi_{5}$ is odd. Also note that the derivative along the fifth dimension of an odd variable is even and vice versa. For instance, $W^{\prime}(y)=W^{\prime}(-y)$. Based on these $Z_{2}$ truncations we should now discuss the continuity properties of all quantities. Normally, one requires the metric to be continuous across the whole of space-time in order to have a sensible notion of length and time. We will also adopt this viewpoint, however with an additional subtlety. Since the orbifolding identifies the upper and lower half of the circle in the fifth dimension already one of them, say the upper half, constitutes the whole of space-time. In fact, instead of working with the orbifold picture where one keeps the full circle as we do here, one could also use the boundary picture where only one half of the circle (a line-segment) is considered. This shows that a jump of a metric component at an orbifold fix point does not contradict the continuity requirement. Of course, such a jump is possible only for an odd component of the metric. Concretely, we therefore require that all components of the metric (47) are continuous across the full orbicircle except for the odd component $W$ which may jump at the fix points (but is continuous otherwise). Corresponding assumptions have to be made for the parameter $\xi^{\alpha}$ so that coordinate transformations do not change these continuity properties of the metric. Clearly the even components $\xi_{0}$ and $\xi$ have to be continuous then. Is the odd component $\xi_{5}$ allowed to jump at the orbifold points? 
Equation (26) shows that $\Gamma=-\phi_{5}^{5}$, which has to be even and continuous, transforms with the derivative $\xi_{5}^{\prime}$. Hence, if $\xi_{5}$ jumped at the fix points it would lead to a delta-function singularity in the metric which is clearly unacceptable. We, therefore, have to require that $\xi_{5}$ is continuous everywhere on the orbicircle. ${ }^{1}$ In particular, this means that $\xi_{5}$ vanishes at the fix points, that is $\xi_{5}\left(y_{n}\right)=0$.

It is clear that the above conclusions depend somewhat on the fact that we are working with an orbifold. For example, if we had considered compactification on a circle instead, all components of the metric had to be continuous. Correspondingly, some of the conclusions below will be slightly modified for other compactifications, however, in a way that is usually rather obvious.

Given this setup the Einstein equations can be written as

$$
G_{\alpha \beta} \equiv R_{\alpha \beta}-\frac{1}{2} g_{\alpha \beta} R=T_{\alpha \beta}+\sum_{n=1}^{2} T_{\alpha \beta}^{(n)} \delta\left(y-y_{n}\right),
$$

where we have set the five-dimensional Newton constant to one, for simplicity. The delta functions in this equation are covariant with respect to the fifth dimension, that is, they include a factor of $1 / \sqrt{-g_{55}}$. Furthermore, $T_{\alpha \beta}$ is the bulk stress-energy tensor induced by fields that propagate in the full five-dimensional space time. The brane stress-energy tensors $T_{\alpha \beta}^{(n)}$, on the other hand, originate from fields that are confined to the branes at the orbifold fix points.

In order to proceed further, we need to specify these stress-energy tensors. Two requirements should be taken into account when doing this. First, one should use the fact that the background has a maximally symmetric threedimensional space. Secondly, the brane stress-energy tensors should be restricted in a way that is consistent with their nature of representing fields on the branes. This latter requirement can be most easily implemented by using the constraints that follow from the Einstein equation (53) itself.
Concretely, the delta functions on the right-hand side of this equation have to be matched by corresponding delta functions that appear on the left-hand side. The appearance of these latter delta functions, however, is controlled by the structure of the equations and the continuity assumptions about the metric discussed above.

Let us see what this implies in detail. We start with the background stress-energy. For the bulk, the most general form of this tensor consistent with the three-dimensional maximal symmetry is

$$
T_{\beta}^{\alpha}=\left(\begin{array}{ccc}
\rho & 0 & -r \\
0 & -p \delta_{j}^{i} & 0 \\
r & 0 & -q
\end{array}\right) .
$$

In particular, we note that the 05 component can be nonvanishing. This possibility is, in fact, already realized for the simple case of a bulk scalar field that depends on $t$ and $y$. As far as the symmetry of the background metric is concerned, the background brane stress-energy tensors should have the same structure as Eq. (54). However, as we will see in a moment, there are two more requirements that follow from the equations of motion, namely that the 55 and the 05 components vanish. As a result, the background stress-energy on the branes has the form

$$
T_{\beta}^{(n) \alpha}=\left(\begin{array}{ccc}
\rho^{(n)} & 0 & 0 \\
0 & -p^{(n)} \delta_{j}^{i} & 0 \\
0 & 0 & 0
\end{array}\right) .
$$

Let us now proceed to the perturbed stress-energy tensors. Since we are focusing on scalar perturbations we write the most general perturbation of the background bulk tensor (54) that can be expressed in terms of scalars on the maximally symmetric subspace. This leads to

$$
\delta T_{\beta}^{\alpha}=\left(\begin{array}{ccc}
\delta \rho & -(\rho+p) b^{-2} v_{\mid j} & -\delta r \\
(\rho+p) v^{\mid i} & -\delta p \delta^{i}+\sigma^{\mid i}{ }_{\mid j} & -u^{\mid i} \\
\delta r+2 r(\phi+\Gamma)-(\rho+q) W & -b^{-2} u_{\mid j} & -\delta q
\end{array}\right)
$$

where $v$ and $u$ are two potentials for "velocity" fields and $\sigma$, satisfying $\sigma^{\mid i}{ }_{i}=0$, determines the anisotropic stress. The perturbed brane stress-energy tensors should have the same structure. However, as we will see below, the equations of motion impose further constraints implying vanishing 55 and $5 i$ components as well as vanishing anisotropic stress. Therefore, the brane stress-energy perturbations are given by

$$
\delta T_{\beta}^{(n) \alpha}=\left(\begin{array}{ccc}
\delta \rho^{(n)} & -\left(\rho^{(n)}+p^{(n)}\right) b^{-2} v_{\mid j}^{(n)} & -\delta r^{(n)} \\
\left(\rho^{(n)}+p^{(n)}\right) v^{(n) \mid i} & -\delta p^{(n)} \delta^{i} & 0 \\
\delta r^{(n)}-\rho^{(n)} W & 0 & 0
\end{array}\right) .
$$

We would like to present the equations of motion based on the metric (47) and on the above stress-energy tensors that follow from the Einstein equation (53). However, in writing the metric (47) two gauge choices were involved and it is not, a priori, clear that these choices can be made while, at the same time, keeping the branes at $y=$ const hypersurfaces as we have

\footnotetext{
${ }^{1}$ From this conclusion we see that we have glossed over a subtlety when introducing the generalized longitudinal gauge. Clearly, for continuous $\xi_{5}$ the quantity $B_{5}$ can only be gauged to zero if $E_{5}-B_{5}$ is continuous, as can be seen from Eqs. (28) and (29). We will, therefore, in addition require the continuity of $E_{5}-B_{5}$. 
conveniently assumed in writing Eq. (53). As the first choice, we decided to work with the two-dimensional metric $\gamma_{a b}$ in conformal gauge. Fortunately, this can be achieved while keeping the branes at hypersurfaces $y=$ const [13]. In addition, we have chosen the generalized longitudinal gauge (37) for the scalar perturbations. A brane, described by $y=y_{n}$ before the gauge transformation that leads to longitudinal gauge, is described by $y=\tilde{y}-\xi^{5}(y)=y_{n}$ after this gauge transformation, where $\tilde{y}$ is the transformed $y$ coordinate. However, since $\xi^{5}\left(y_{n}\right)=0$, as discussed above, this equation is solved by $\tilde{y}=y_{n}$ to linear order. We conclude that, in the new coordinates that lead to the generalized longitudinal gauge, the brane location is unchanged to the relevant linear order. In summary, therefore, using the Einstein equation (53) with the branes described by $y=y_{n}$ does not restrict the generality of our results given the gauge choices that we have made.

The background equations following from Eq. (53) have been given in Ref. [13] for the case of stress-energy induced by scalar fields and in Ref. [15] for the case of ideal fluids. For completeness and in order to incorporate some of the generalizations that we have made (such as the inclusion of a 05 component of the bulk stress-energy) we will nevertheless present these equations here. We find

$$
\begin{aligned}
& a^{2} b^{2} G_{0}^{0} \equiv 3\left[2 \frac{\dot{a}^{2}}{a^{2}}+\frac{\dot{a} \dot{b}}{a b}-\frac{a^{\prime \prime}}{a}+\frac{a^{\prime} b^{\prime}}{a b}+k b^{2}\right]=a^{2} b^{2}\left[\rho+\sum_{n=1}^{2} \rho^{(n)} \bar{\delta}\left(y-y_{n}\right)\right] \\
& a^{2} b^{2} G^{5}{ }_{5} \equiv 3\left[\frac{\ddot{a}}{a}-\frac{\dot{a} \dot{b}}{a b}-2 \frac{a^{\prime 2}}{a^{2}}-\frac{a^{\prime} b^{\prime}}{a b}+k b^{2}\right]=-a^{2} b^{2} q \\
& a^{2} b^{2} G^{0}{ }_{5} \equiv 3\left[-\frac{\dot{a}^{\prime}}{a}+2 \frac{\dot{a} a^{\prime}}{a^{2}}+\frac{\dot{a} b^{\prime}}{a b}+\frac{a^{\prime} \dot{b}}{a b}\right]=-a^{2} b^{2} r \\
& a^{2} b^{2} G_{j}^{i} \equiv\left[3 \frac{\ddot{a}}{a}+\frac{\ddot{b}}{b}-\frac{\dot{b}^{2}}{b^{2}}-3 \frac{a^{\prime \prime}}{a}-\frac{b^{\prime \prime}}{b}+\frac{b^{\prime 2}}{b^{2}}+k b^{2}\right] \delta_{j}^{i}=-a^{2} b^{2}\left[p+\sum_{n=1}^{2} p^{(n)} \bar{\delta}\left(y-y_{n}\right)\right] \delta_{j}^{i} .
\end{aligned}
$$

Here we have defined the delta-function $\bar{\delta}$ which incorporates a factor $1 / a b$. Based on these equations we can now justify the vanishing of the 55 and 05 components in the brane stress-energy (55). Such components, if non-vanishing, would appear on the right-hand sides of Eqs. (59) and (60) multiplied with delta-functions. We should, therefore, have corresponding deltafunction terms on the left-hand sides of these equations. Since the scale factors $a$ and $b$ are assumed to be continuous, delta functions can only appear from second derivatives of these quantities with respect to $y$. However, there are no such terms in Eqs. (59) and (60). Hence, we conclude that the 55 and 05 components in Eq. (55) must vanish.

For the perturbations, we find, to linear order,

$$
\begin{aligned}
(a b)^{2} \delta G_{0}^{0}{ }_{0}= & 3\left[2 \frac{a^{\prime} b^{\prime}}{a b}-2 \frac{a^{\prime \prime}}{a}-\frac{a^{\prime}}{a} \frac{\partial}{\partial y}-\frac{\dot{a}}{a} \frac{\partial}{\partial t}\right] \Gamma-3\left[\frac{\dot{a}^{\prime}}{a}+2 \frac{a^{\prime} \dot{a}}{a^{2}}+\frac{\dot{a}}{a} \frac{\partial}{\partial y}\right] W-6\left[2 \frac{\dot{a}^{2}}{a^{2}}+\frac{\dot{a} \dot{b}}{a b}\right] \phi \\
& +3\left[3 \frac{a^{\prime}}{a} \frac{\partial}{\partial y}-\frac{b^{\prime}}{b} \frac{\partial}{\partial y}-3 \frac{\dot{a}}{a} \frac{\partial}{\partial t}-\frac{\dot{b}}{b} \frac{\partial}{\partial t}+2 k b^{2}\right] \psi+b^{2}(2 \psi+\Gamma)^{\mid i}{ }_{i}+3 \psi^{\prime \prime} \\
= & a^{2} b^{2}\left\{\delta \rho+\sum_{n=1}\left(\delta \rho^{(n)}+\Gamma \rho^{(n)}\right) \bar{\delta}\left(y-y_{n}\right)\right\} \\
(a b)^{2} \delta G_{5}^{5} \equiv & -6\left[2 \frac{a^{\prime 2}}{a^{2}}+\frac{a^{\prime} b^{\prime}}{a b}\right] \Gamma-3\left[\frac{a^{\prime}}{a}+2 \frac{a^{\prime} \dot{a}}{a^{2}}+\frac{a^{\prime}}{a} \frac{\partial}{\partial t}\right] W+3\left[2 \frac{\dot{a} \dot{b}}{a b}-2 \frac{\ddot{a}}{a}-\frac{a^{\prime}}{a} \frac{\partial}{\partial y}-\frac{\dot{a}}{a} \frac{\partial}{\partial t}\right] \phi \\
& +3\left[3 \frac{a^{\prime}}{a} \frac{\partial}{\partial y}+\frac{b^{\prime}}{b} \frac{\partial}{\partial y}-3 \frac{\dot{a}}{a} \frac{\partial}{\partial t}+\frac{\dot{b}}{b} \frac{\partial}{\partial t}+2 k b^{2}\right] \psi+b^{2}(2 \psi-\phi)^{\mid i}-3 \ddot{\psi} \\
= & -a^{2} b^{2} \delta q
\end{aligned}
$$




$$
\begin{aligned}
& (a b)^{2} \delta G^{0}{ }_{5} \equiv 3\left[\frac{a^{\prime \prime}}{a}-2 \frac{a^{\prime} b^{\prime}}{a b}-2 \frac{a^{\prime 2}}{a^{2}}\right] W+3\left[2 \frac{\dot{a}^{\prime}}{a}-2 \frac{\dot{a} b^{\prime}}{a b}-2 \frac{a^{\prime} \dot{b}}{a b}-4 \frac{a^{\prime} \dot{a}}{a^{2}}+\frac{\dot{a}}{a} \frac{\partial}{\partial y}\right] \phi \\
& +3\left[\frac{\partial^{2}}{\partial t \partial y}-\frac{b^{\prime}}{b} \frac{\partial}{\partial t}-\frac{\dot{b}}{b} \frac{\partial}{\partial y}\right] \psi-\frac{b^{2}}{2} W^{\mid i}{ }_{\mid i}-3 \frac{a^{\prime}}{a} \dot{\Gamma} \\
& =a^{2} b^{2}\left\{-\delta r-\sum_{n=1}^{2} \delta r^{(n)} \bar{\delta}\left(y-y_{n}\right)\right\} \\
& (a b)^{2} \delta G_{i}^{0} \equiv\left\{\left[\frac{3}{2} \frac{a^{\prime}}{a}+\frac{b^{\prime}}{b}+\frac{1}{2} \frac{\partial}{\partial y}\right] W+\left[3 \frac{\dot{a}}{a}+\frac{\dot{b}}{b}\right] \phi+\left[\frac{\dot{b}}{b}+\frac{\partial}{\partial t}\right] \Gamma+2 \dot{\psi}\right\}_{\mid i} \\
& =a^{2}\left\{-(\rho+p) v-\sum_{n=1}^{2}\left(\rho^{(n)}+p^{(n)}\right) v^{(n)} \bar{\delta}\left(y-y_{n}\right)\right\}_{\mid i} \\
& (a b)^{2} \delta G_{i}^{5} \equiv\left\{\left[3 \frac{a^{\prime}}{a}+\frac{b^{\prime}}{b}\right] \Gamma+\left[\frac{b^{\prime}}{b}+\frac{\partial}{\partial y}\right] \phi+\left[\frac{3}{2} \frac{\dot{a}}{a}+\frac{\dot{b}}{b}+\frac{1}{2} \frac{\partial}{\partial t}\right] W-2 \psi^{\prime}\right\}_{\mid i}=-a^{2} u_{\mid i} \\
& (a b)^{2} \delta G_{j}^{i} \equiv\left\{\left[-6 \frac{a^{\prime \prime}}{a}-2 \frac{b^{\prime \prime}}{b}+2 \frac{b^{\prime 2}}{b^{2}}-3 \frac{a^{\prime}}{a} \frac{\partial}{\partial y}-3 \frac{\dot{a}}{a} \frac{\partial}{\partial t}-\frac{\dot{b}}{b} \frac{\partial}{\partial t}-\frac{b^{\prime}}{b} \frac{\partial}{\partial y}-\frac{\partial^{2}}{\partial t^{2}}\right] \Gamma\right. \\
& +\left[2 \frac{b^{\prime} \dot{b}}{b^{2}}-2 \frac{\dot{b}^{\prime}}{b}-6 \frac{\dot{a}^{\prime}}{a}-3 \frac{a^{\prime}}{a} \frac{\partial}{\partial t}-3 \frac{\dot{a}}{a} \frac{\partial}{\partial y}-\frac{\dot{b}}{b} \frac{\partial}{\partial y}-\frac{b^{\prime}}{b} \frac{\partial}{\partial t}-\frac{\partial^{2}}{\partial t \partial y}\right] W \\
& +\left[2 \frac{\dot{b}^{2}}{b^{2}}-2 \frac{\ddot{b}}{b}-6 \frac{\ddot{a}}{a}-3 \frac{a^{\prime}}{a} \frac{\partial}{\partial y}-3 \frac{\dot{a}}{a} \frac{\partial}{\partial t}-\frac{\dot{b}}{b} \frac{\partial}{\partial t}-\frac{b^{\prime}}{b} \frac{\partial}{\partial y}-\frac{\partial^{2}}{\partial y^{2}}\right] \phi+\left[6 \frac{a^{\prime}}{a} \frac{\partial}{\partial y}-6 \frac{\dot{a}}{a} \frac{\partial}{\partial t}+2 \frac{\partial^{2}}{\partial y^{2}}-2 \frac{\partial^{2}}{\partial t^{2}}\right] \psi \\
& \left.+2 b^{2}(\psi-\phi+\Gamma)^{\mid k} \mid k\right\} \delta_{j}^{i}-b^{2}(\psi-\phi+\Gamma)^{\mid i}{ }_{\mid j} \\
& =a^{2} b^{2}\left\{-\delta p \delta_{j}^{i}+\sigma^{\mid i}{ }_{\mid j}-\sum_{n=1}^{2}\left(\delta p^{(n)}+\Gamma p^{(n)}\right) \delta_{j}^{i} \bar{\delta}\left(y-y_{n}\right)\right\} .
\end{aligned}
$$

Given those results, we can now return to the question of why the perturbations of the brane stress-energy tensors must have the specific form (57). We recall that all quantities in our metric (47) are even except the off-diagonal perturbation $W$ which is odd under the $Z_{2}$ symmetry. From our continuity assumptions, delta function terms in the perturbed Einstein tensor can, therefore, arise from first derivatives of $W$ with respect to $y$ and second derivatives with respect to $y$ of all other quantities. Inspection of the above equations shows that such terms are absent in the $5 i$ and 55 components of the perturbed Einstein tensor. Consequently, the corresponding components in the perturbed brane stress-energy should vanish. Furthermore, all terms in $\delta G^{i}{ }_{j}$ that could potentially lead to delta-functions are proportional to $\delta^{i}{ }_{j}$. This implies that the anisotropic stress on the brane, which would contribute to the traceless part of Eq. (67), must vanish. As a result, the traceless part of Eq. (67)

$$
(\psi-\phi+\Gamma)^{\mid i}{ }_{\mid j}-\frac{1}{3}(\psi-\phi+\Gamma)^{\mid k}{ }_{\mid k} \delta_{j}^{i}=-a^{2} \sigma^{\mid i}{ }_{\mid j}
$$

only involves the bulk anisotropic stress as a source term. If the bulk anisotropic stress vanishes as well, as, for example, is the case for a perfect fluid in the bulk, one concludes that

$$
\psi-\phi+\Gamma=0
$$

The quantity $\psi-\phi+\Gamma$ is the analog of the four-dimensional quantity $\psi_{4}-\phi_{4}$ that also vanishes in the absence of anisotropic stress. This correspondence will be made more explicit in Sec. V.

\section{DENSITY FLUCTUATIONS ON THE BRANE}

A systematic study of density fluctuation in five dimensions requires solving the full set of five-dimensional equations of motion given in the previous section. However, for specific questions it might be useful to have some information about the metric restricted to the brane. For example, it is this restricted metric that is felt by matter which is con- 
fined to the brane. In this section, we are going to derive such equations on the brane starting from the general equations of motion above.

For a $Z_{2}$ even field the meaning of its value on the brane is quite clear. A $Z_{2}$ odd field may jump across the brane so its value may have a sign ambiguity. To simplify the notation, we define the value of an odd field on the brane as the one that is approached from within the interval $y \in[0, R]$. This is precisely the boundary value of the field as viewed in the boundary picture and, at the same time represents one half of the jump at the fix point. We also recall that the scale factors $a, b$ and the perturbations $\phi, \psi$ and $\Gamma$ are even and hence continuous while the perturbation $W$ is odd and may jump across the fix points.

Let us start with the background equations of motion $[13,15]$. We have already mentioned that the delta-function sources in Eqs. (58) and (61) have to be matched by the terms containing second $y$ derivatives of the scale factors $a$ and $b$. This leads to

$$
\frac{a^{\prime}}{a}=\mp \frac{1}{6} a b \rho^{(n)}, \quad \frac{b^{\prime}}{b}= \pm \frac{1}{2} a b\left(\rho^{(n)}+p^{(n)}\right) .
$$

These conditions, as well as the following ones, are valid at the brane positions $y=y_{n}$ where the upper (lower) sign holds for the brane $n=1 \quad(n=2)$. While the two other nonvanishing equations of motion do not contain delta functions, they can still be restricted to the brane. From the 05 component (60) we find

$$
\dot{\rho}^{(n)}=-3 \frac{\dot{a}}{a}\left(\rho^{(n)}+p^{(n)}\right) \mp 2 a b r
$$

which represents to an energy conservation equation on the brane. Note, however, that, in addition to intrinsic brane quantities, this equation also involves the off-diagonal bulk stress-energy component $r$. This reflects the simple fact that the branes are not isolated systems but can exchange energy with the bulk. Finally, we should consider the 55 component (59). Restricted to the branes it results in an equation of motion for the values of the scale factors $a$ and $b$ on the brane given by

$$
\frac{\ddot{a}}{a}-\frac{\dot{a} \dot{b}}{a b}+k b^{2}=-\frac{a^{2} b^{2}}{3}\left[\frac{1}{12} \rho^{(n)}\left(\rho^{(n)}+3 p^{(n)}\right)+q\right] .
$$

An analogous procedure can now be applied to the perturbed equations. Observe that only the components $\delta G_{0}^{0}, \delta G_{i}^{i}$, $\delta G_{5}^{0}$ and $\delta G_{i}^{0}$ contain explicit delta-function terms. They should be matched by terms containing first $y$ derivatives of $W$ and second $y$ derivatives of all other quantities. This leads to

$$
\psi^{\prime}=\frac{\dot{a}}{a} W \pm \frac{1}{6} a b\left(\delta \rho^{(n)}-\Gamma \rho^{(n)}\right)
$$

$$
\begin{aligned}
\phi^{\prime}= & -\left(\frac{\dot{a}}{a}+\frac{\dot{b}}{b}+\frac{\partial}{\partial t}\right) W \pm \frac{1}{3} a b\left(\delta \rho^{(n)}-\Gamma \rho^{(n)}\right) \\
& \pm \frac{1}{2} a b\left(\delta p^{(n)}-\Gamma p^{(n)}\right) \\
W= & \mp \frac{a}{b}\left(\rho^{(n)}+p^{(n)}\right) v^{(n)} \\
W= & \frac{\delta r^{(n)}}{\rho^{(n)}} .
\end{aligned}
$$

Interestingly, the last equation implies that the component $\delta T^{(n) 5}{ }_{0}$ of the brane stress-energy perturbation vanishes, as can be seen by comparison with Eq. (57). The component $\delta T^{(n) 0}{ }_{5}=-\delta r^{(n)}$, however, is non-zero and is, from Eqs. (75), (76) determined by

$$
\delta r^{(n)}=\mp \frac{a}{b} \rho^{(n)}\left(\rho^{(n)}+p^{(n)}\right) v^{(n)} .
$$

We have, therefore, found an important additional constraint on the perturbed brane energy-momentum tensor (57). The quantity $\delta r^{(n)}$ is, in fact, uniquely fixed by the other components. For vacuum energy with $p^{(n)}=-\rho^{(n)}$ on the branes $\delta r^{(n)}$ is zero, but it is generally non-vanishing otherwise. This is, perhaps, somewhat surprising since one could have expected that a purely four-dimensional stress-energy tensor on the brane (with all 5 components vanishing) should be allowed. Here we see that this is generally not the case.

Next, we deal with the odd components 05 and $5 i$ of the perturbed equations of motion given in Eqs. (64) and (66). Restriction to the branes leads us, after some algebra, to

$$
\begin{aligned}
\dot{\delta}^{(n)}= & -\left(1+w^{(n)}\right)\left(v^{(n) \mid i} \mid i-3 \dot{\psi}\right)-3 \frac{\dot{a}}{a}\left(\frac{\delta p^{(n)}}{\delta \rho^{(n)}}-w^{(n)}\right) \delta^{(n)} \\
& -2\left(1+w^{(n)}\right) v^{(n)} a^{2}(\rho+q) \\
& \mp 2 a b\left(\Gamma+2 \phi-\delta^{(n)}+\frac{\delta r}{r}\right) \frac{r}{\rho^{(n)}}
\end{aligned}
$$

and

$$
\begin{aligned}
\frac{\dot{v}^{(n)} \mid i}{b^{2}}= & {\left[-\frac{\dot{a}}{a}\left(1-3 w^{(n)}\right)-\frac{\dot{w}^{(n)}}{1+w^{(n)}}+\frac{\dot{b}}{b}\right] \frac{v^{(n)} \mid i}{b^{2}} } \\
& -\frac{\delta p^{(n)} \mid i}{\delta \rho^{(n)}} \frac{\delta^{(n)}}{1+w^{(n)}}-\phi_{\mid i} \\
& \mp 2 \frac{a}{b} \frac{1}{\rho^{(n)}}\left[\frac{u}{1+w^{(n)}}-r v^{(n)}\right]_{\mid i},
\end{aligned}
$$

where $w^{(n)}=p^{(n)} / \rho^{(n)}$ and $\delta^{(n)}=\delta \rho^{(n)} / \rho^{(n)}$ denotes the energy contrast on the branes. These equations represent the conservation of energy and momentum for scalar perturbations, including possible energy-momentum flow from the 
bulk onto the brane or from the brane into the bulk. They should be compared with the corresponding equations in four dimensions, Eq. (30) in [51], taking into account that the variable $\theta$ of [51] is related to the peculiar velocity $v$ via $\theta$ $=v^{\mid i}{ }_{i}$. Equation (78) differs from the four-dimensional result by the last two terms. They describe the energy flux from the bulk onto the brane. In Eq. (79) we have two new terms with respect to the four-dimensional equation. They describe momentum flux between bulk and brane. The coupling between bulk gravity and brane matter expressed via the above equations is one of the main results of this paper. It shows that, when considering scalar metric perturbations on the branes, the branes cannot simply be viewed as an isolated system but have to be considered together with the bulk environment. Practically, this implies that frequently one cannot simply copy four-dimensional formulas when dealing with physics on a brane that is embedded in a higherdimensional space. Finally, we restrict the 55 component of the equations of motion, Eq. (63), to the brane. We find the following evolution equation for the perturbations projected onto the branes:

$$
\begin{aligned}
& b^{2}(2 \psi-\phi)^{\mid i}{ }_{i i}-3 \ddot{\psi}-3 \frac{\dot{a}}{a} \dot{\phi}+3\left(\frac{\dot{b}}{b}-3 \frac{\dot{a}}{a}\right) \dot{\psi}+6 k b^{2}(\psi+\phi) \\
& +a^{2} b^{2} \rho^{(n) 2}\left[\frac{1}{6}\left(1+3 w^{(n)}\right) \phi+\frac{\delta q+2 q \phi}{\rho^{(n) 2}}\right. \\
& +\frac{1}{6}\left(1+\frac{3}{2} w^{(n)}\right) \delta^{(n)}+\frac{\delta p^{(n)}}{4 \delta \rho^{(n)}} \delta^{(n)} \\
& \left. \pm \frac{a}{b} \frac{r}{\rho^{(n)}}\left(1+w^{(n)}\right) v^{(n)}\right]=0 .
\end{aligned}
$$

\section{MATCHING TO THE FOUR-DIMENSIONAL EFFECTIVE THEORY}

In the previous subsection, we have derived a set of equations for the metric on the branes, essentially by restricting the five-dimensional equations of motion. These results may, for example, be useful to analyze the evolution of matter that is confined to the brane. However, the most important task is to extract predictions for structure in the late universe from our formalism of metric perturbations in brane-world theories. In this section, we will explain the basic steps in this direction.

First, we should introduce the four-dimensional effective theory, describing physics at low energy, that is associated to our five-dimensional brane-world theory (53). It is this fourdimensional theory that described the evolution of the universe "today" and that is used for the interpretation of observational results. Theoretical predictions, originating from our brane-world theory, should therefore be formulated in terms of this effective theory. The five- and the fourdimensional effective theory are related by a vacuum state that constitutes a specific solution of the five-dimensional theory and should respect the symmetries that we expect to find in the four-dimensional theory. Specifically, four- dimensional Lorentz invariance implies that the vacuum metric should have the structure

$$
d s^{2}=A^{2}(y) d x^{\mu} d x^{\nu} \eta_{\mu \nu}-B^{2}(y) d y^{2} .
$$

The functions $A$ and $B$ have to be such that this metric solves the five-dimensional theory in the vacuum configuration. For our five-dimensional theory (53), the simplest possibility is to have no stress energy in the vacuum which results in a flat vacuum metric, $A, B=$ const. In five-dimensional heterotic $\mathrm{M}$ theory the vacuum configuration is determined by certain potentials in the bulk and on the branes that involve the dilaton $[4,39]$. In this case, $A$ and $B$ are non-trivial functions of $y$ and the deviation from the flat vacuum metric is determined by the size of the so called strong coupling expansion parameter. The vacua proposed in Refs. [41,42] are based on a vacuum configuration with specific cosmological constants in the bulk and on the branes and result in an exponential function for $A$ in the coordinate system where $B=$ const. Each one of these different vacuum states is associated with its specific low-energy theory. For the sake of simplicity and concreteness, we will here focus on the first possibility, namely the flat vacuum. This choice represents, at the same time, a good approximation for five-dimensional heterotic $\mathrm{M}$ theory in the case of a small strong-coupling expansion parameter. The four-dimensional effective theory describes the dynamics of the collective excitations of the vacuum state. In our case, these excitation are given by a four-dimensional metric $g_{4 \mu \nu}$ and the modulus $\beta$ describing the size of the fifth dimension. The vacuum metric with these collective modes put in has the structure

$$
d \bar{s}^{2}=e^{-\beta} g_{4 \mu \nu} d x^{\mu} d x^{\nu}-e^{2 \beta} d y^{2},
$$

where $g_{4 \mu \nu}$ and $\beta$ are functions of $x^{\mu}$. As usual, the effective four-dimensional description is valid as long as these functions are varying slowly enough. This is the case if all fourdimensional momenta are much smaller than the mass of the first Kaluza-Klein excitation around the vacuum state. In our case this mass is given by $e^{-\beta} / 2 R$. Let us, therefore, consider a five-dimensional evolution that is approaching the vacuum state (82). Even though the five-dimensional metric is then close to the vacuum metric it will still have small Kaluza-Klein excitations that can be described in linear perturbation theory. A useful way to extract the zero modes from such a five-dimensional metric with small contributions from Kaluza-Klein modes is to perform an average over the fifth dimension. Doing this systematically leads to the following four-dimensional effective theory associated to the brane-world theory (53) and the vacuum state (82):

$$
\begin{aligned}
R_{4 \mu \nu}-\frac{1}{2} g_{4 \mu \nu} R_{4} & =\frac{3}{2}\left(\partial_{\mu} \beta \partial_{\nu} \beta-\frac{1}{2} g_{4 \mu \nu} \partial \beta^{2}\right)+T_{4 \mu \nu} \\
\nabla_{4}^{2} \beta & =J_{4} .
\end{aligned}
$$

Four- and five-dimensional quantities are related by

$$
e^{2 \beta}=-\left\langle g_{55}\right\rangle
$$




$$
\begin{aligned}
g_{4 \mu \nu} & =e^{\beta}\left\langle g_{\mu \nu}\right\rangle \\
T_{4 \mu \nu} & =\left\langle T_{\mu \nu}\right\rangle+\frac{1}{2 \operatorname{Re}^{\beta}} \sum_{n=1}^{2} T_{\mu \nu}^{(n)} \\
J_{4} & =\frac{2}{3} e^{-3 \beta}\left\langle T_{55}\right\rangle+\frac{1}{3} g_{4}^{\mu \nu} T_{4 \mu \nu} .
\end{aligned}
$$

Here, $\langle\cdot\rangle$ denotes the average over the fifth dimension. As stated, this four-dimensional theory is a good description as long as all momenta are small compared to $e^{-\beta} / 2 R$, the mass of the first Kaluza-Klein mode. The Kaluza-Klein modes have decoupled from the above equations at linear order. However, due to the presence of the branes, the Kaluza-Klein modes cannot strictly be set to zero but have to be integrated out $[52,53]$. This leads to higher order corrections to the above four-dimensional equations that are suppressed by powers of the four-dimensional Planck scale and that we have neglected. To be consistent with this approximation, the average $\langle\cdot\rangle$ that projects out the Kaluza-Klein excitations should be considered meaningful only at the linearized level in Kaluza-Klein excitations.

We would now like to apply the above general correspondence to our formalism for metric fluctuations. To do this, we need to assume a five-dimensional background solution that, for late time, approaches the vacuum configuration. Formulated in a four-dimensional language, this is the case if the four-dimensional Hubble parameter $H_{4}=\dot{a}_{4} / a_{4}$ and $\dot{\beta}$ are small compared to $e^{-\beta} / 2 R$. Furthermore, it is helpful to assume that the average $\left\langle\gamma_{00}\right\rangle$ approaches one in this limit. This can always be achieved by a redefinition of time. We would like to explicitly work out the correspondence for the scalar sector in longitudinal gauge that we have focused on in this paper. The generalization to include vector and tensor perturbations is straightforward. Concretely, we apply the correspondence $(85)-(88)$ to the five-dimensional quantities (47), (54), (55), (56) and (57) matching onto the fourdimensional quantities specified in Eqs. (18), (19) and (20). Furthermore, we need to decompose the four-dimensional modulus $\beta$ as

$$
\beta=\chi-\Gamma_{4}
$$

where $\chi=\chi(t)$ is the time-dependent background and $\Gamma_{4}$ $=\Gamma_{4}\left(t, x^{i}\right)$ is the perturbation. The matching of background quantities leads to

$$
\begin{aligned}
e^{2 \chi} & =\left\langle a^{2} b^{2}\right\rangle \\
a_{4}^{2} & =e^{\chi}\left\langle a^{2}\right\rangle \\
\rho_{4} & =e^{-\chi}\langle\rho\rangle+\frac{1}{2 \operatorname{Re}^{2 \chi}} \sum_{n=1}^{2} \rho^{(n)} \\
p_{4} & =e^{-\chi}\langle p\rangle+\frac{1}{2 \operatorname{Re}^{2 \chi}} \sum_{n=1}^{2} p^{(n)} .
\end{aligned}
$$

It is interesting to explicitly compute the background current $J_{4}$ in the equation of motion (84) for the modulus $\chi$. It is given by

$$
J_{4}=\frac{1}{3}\left[\rho_{4}-3 p_{4}+2 e^{-\chi}\langle q\rangle\right]
$$

With the above expression for $\rho_{4}$ and $p_{4}$, this implies that the modulus $\chi$ has a runaway potential leading to a growing size of the fifth dimension. Therefore, the theory, as stands, will not stay in the range of validity of the four-dimensional effective theory. As is well-known, it needs further stabilization of the modulus $\beta$ by means of a potential. In the context of string or $\mathrm{M}$ theory one expects such a potential to be generated by non-perturbative effects.

The correspondence for the scalar perturbations reads

$\Gamma_{4}=\langle\Gamma\rangle$

$\phi_{4}=\langle\phi-\Gamma / 2\rangle$

$\psi_{4}=\langle\psi+\Gamma / 2\rangle$

$\delta \rho_{4}=e^{-\chi}\langle\delta \rho+\Gamma \rho\rangle+\frac{1}{2 \operatorname{Re}^{2 \chi}} \sum_{n=1}^{2}\left(\delta \rho^{(n)}+2 \Gamma_{4} \rho^{(n)}\right)$

$\delta p_{4}=e^{-\chi}\langle\delta p+\Gamma p\rangle+\frac{1}{2 \operatorname{Re}^{2 \chi}} \sum_{n=1}^{2}\left(\delta p^{(n)}+2 \Gamma_{4} p^{(n)}\right)$

$\sigma_{4}=e^{-\chi}\langle\sigma\rangle$

$v_{4}=\frac{e^{-3 \chi} a_{4}^{4}}{\rho_{4}+p_{4}}\left[\langle(\rho+p) v\rangle+\frac{1}{2 \operatorname{Re}^{\beta}} \sum_{n=1}^{2}\left(\rho^{(n)}+p^{(n)}\right) v^{(n)}\right]$.

In particular, we conclude that

$$
\psi_{4}-\phi_{4}=\langle\psi-\phi+\Gamma\rangle \text {. }
$$

Hence, the four- and five-dimensional quantities that measure the presence of anisotropic stress are in direct correspondence to one another as they should.

\section{CONCLUSION}

In this paper we have laid down a gauge-invariant formalism to describe metric fluctuations in brane-world theories. This formalism is a straightforward generalization of the well known formalism in four dimensions. It categorizes the perturbations according to their tensor properties with respect to the usual three-dimensional maximally symmetric space rather than a higher-dimensional space as one might have expected. This is a direct consequence of the braneworld nature of the theory which generally leads to cosmological backgrounds that are inhomogeneous in the additional dimensions. We have introduced a generalized longitudinal gauge in order to further study scalar perturba- 
tions. In the case of a five-dimensional model on the orbifold $S^{1} / Z_{2}$, on which we have focused, we have identified four scalar metric perturbations $\phi, \psi, \Gamma$ and $W$. This has to be contrasted to the four-dimensional case where one only has two such perturbations. We have presented the evolution equations for these scalar perturbations which, mainly due to the dependence of the background on the additional dimension, are significantly more complicated than the corresponding four-dimensional equations. It is those additional terms, related to the non-linearity of the background in the additional coordinates, that encode possible new and interesting information about the formation and evolution of perturbations. Furthermore, given the gauge choices and assumptions about the continuity of the metric that we have made, we have determined the resulting most general form of the stress energy on the brane. In particular, we have found that the perturbed brane stress-energy has to have vanishing anisotropic stress and that its 05 component is non-zero. We have applied our formalism to calculate the matching conditions (Israel conditions) for the five-dimensional metric restricted to the branes. Among other results we have derived the equations describing energy and momentum conservation for metric perturbations on the brane. As is expected on physical grounds, they illustrate that the brane cannot be viewed as an isolated object but is subject to energy and momentum flow between the bulk and the brane. Finally, we have shown how the five-dimensional formalism for metric fluctuations can be matched to the known four-dimensional one in the limit where the brane-world theory has an effective fourdimensional description. This allows one to extract predictions for structure in the late universe originating from braneworld theories. We hope to address this problem more explicitly in a future publication.

Note added. As this manuscript was prepared for submission, Refs. [54], [55] and [56] appeared which have some overlap with the present paper. Cosmological perturbations are also discussed in [57].

\section{ACKNOWLEDGMENTS}

C.v.d.B. is grateful to E. Eyras, J. Martin, C. Martins, H. Reall and T. Shiromizu, and in particular D. Langlois, R. Maartens and D. Wands for useful discussions. C.v.d.B. is supported by Nato/DAAD (at Brown) and Deutsche Forschungsgemeinschaft (DFG, at Cambridge). M.D. is supported by the Fundación Ramón Areces. The research was supported in part (at Brown) by the U.S. Department of Energy under Contract DE-FG02-91ER40688, TASK A.A.L. is supported by the European Community under contract No. FMRXCT 960090.
[1] P. Hořava and E. Witten, Nucl. Phys. B460, 506 (1996); B475, 96 (1996).

[2] E. Witten, Nucl. Phys. B471, 135 (1996).

[3] P. Hořava, Phys. Rev. D 54, 7561 (1996).

[4] A. Lukas, B. A. Ovrut, K. S. Stelle, and D. Waldram, Phys. Rev. D 59, 086001 (1999).

[5] N. Arkani-Hamed, S. Dimopolous, and G. Dvali, Phys. Lett. B 429, 263 (1998).

[6] I. Antoniadis, N. Arkani-Hamed, S. Dimopolous, and G. Dvali, Phys. Lett. B 436, 257 (1998).

[7] Z. Kakushadze and S. H. H. Tey, Nucl. Phys. B548, 180 (1999).

[8] J. D. Lykken, Phys. Rev. D 54, 3693 (1996).

[9] A. Lukas, B. A. Ovrut, and D. Waldram, Phys. Rev. D 60, 086001 (1999).

[10] N. Arkani-Hamed, S. Dimopolous, and G. Dvali, Phys. Rev. D 59, 086004 (1999).

[11] H. Reall, Phys. Rev. D 59, 103506 (1999).

[12] A. Lukas, B. A. Ovrut, and D. Waldram, Lectures given at the Advanced School on Cosmology and Particle Physics, 1998, hep-th/9812052, and references therein.

[13] A. Lukas, B. A. Ovrut, and D. Waldram, Phys. Rev. D 61, 023506 (2000).

[14] H. Chamblin and H. Reall, Nucl. Phys. B562, 133 (1999).

[15] P. Binetruy, C. Deffayet, and D. Langlois, Nucl. Phys. B565, 269 (2000).

[16] N. Kaloper, Phys. Rev. D 60, 123506 (1999).

[17] C. Csaki, M. Graesser, C. Kolda, and J. Terning, Phys. Lett. B 462, 34 (1999).

[18] J. Cline, C. Grojean, and G. Servant, Phys. Rev. Lett. 83, 4245 (1999).
[19] D. J. H. Chung and K. Freese, Phys. Rev. D 61, 023511 (2000).

[20] P. J. Steinhardt, Phys. Lett. B 462, 41 (1999).

[21] H. B. Kim and H. D. Kim, Phys. Rev. D 61, 064003 (2000).

[22] U. Ellwanger, Phys. Lett. B 473, 233 (2000).

[23] O. De Wolfe, D. Freedman, S. Gubser, and A. Karch, Phys. Rev. D 62, 046008 (2000).

[24] E. E. Flanagan, S.-H. Tye, and I. Wasserman, Phys. Rev. D 62, 024011 (2000).

[25] P. Kanti, I. I. Kogan, K. A. Olive, and M. Pospelov, Phys. Lett. B 468, 31 (1999).

[26] T. Shiromizu, K.-I. Maeda, and M. Sasaki, Phys. Rev. D 62, 024012 (2000).

[27] P. Binetruy, C. Deffayet, U. Ellwanger, and D. Langlois, Phys. Lett. B 477, 285 (2000).

[28] D. Vollick, "Cosmology on a Three-Brane," hep-th/9911181.

[29] C. Csaki, M. Graesser, L. Randall, and J. Terning, Phys. Rev. D 62, 045015 (2000).

[30] D. Ida, J. High Energy Phys. 09, 014 (2000).

[31] P. Kanti, I. I. Kogan, K. A. Olive, and M. Pospelov, Phys. Rev. D 61, 106004 (2000).

[32] R. Maartens, D. Wands, B. A. Bassett, and I. Heard, Phys. Rev. D 62, 041301(R) (2000).

[33] U. Ellwanger, "Cosmological Evolution in Compactified Hořava-Witten Theory Induced by Matter on the Branes,', hep-th/0001126.

[34] S. W. Hawking, T. Hertog, and H. S. Reall, Phys. Rev. D 62, 043501 (2000).

[35] R. N. Mohapatra, A. Pěrez-Lorenzana, and C. A. de S. Pires, "Cosmology of Brane-Bulk Models in Five Dimensions," hep-ph/0003328. 
[36] N. Deruelle and T. Doležel, Phys. Rev. D 62, 103502 (2000).

[37] C. Barcelo and M. Visser, Phys. Lett. B 482, 183 (2000).

[38] J. Ellis, Z. Lalak, S. Pokorski, and W. Pokorski, Nucl. Phys. B540, 149 (1999).

[39] A. Lukas, B. A. Ovrut, K. S. Stelle, and D. Waldram, Nucl. Phys. B552, 246 (1999).

[40] M. Visser, Phys. Lett. 159B, 22 (1985).

[41] L. Randall and R. Sundrum, Phys. Rev. Lett. 83, 3370 (1999).

[42] L. Randall and R. Sundrum, Phys. Rev. Lett. 83, 4670 (1999).

[43] J. Lykken and L. Randall, J. High Energy Phys. 06, 014 (2000).

[44] J. Bardeen, Phys. Rev. D 22, 1882 (1980).

[45] V. F. Mukhanov, H. A. Feldman, and R. H. Brandenberger, Phys. Rep. 215, 203 (1992).

[46] W. Israel, Nuovo Cimento Soc. Ital. Fis., B 44, 1 (1996).

[47] J. C. Fabris and M. Sakellariadou, Class. Quantum Grav. 14, 725 (1997).

[48] Y. Kubyshin and J. Martin, “Limits on Kaluza-Klein Models from COBE Results," gr-qc/9507010; “'On Compatibility of the Kaluza-Klein Approach with the COBE Experiment," gr-qc/9604031.

[49] R. B. Abbott, B. Bednarz, and S. D. Ellis, Phys. Rev. D 33, 2147 (1986).

[50] R. Burstein, M. Gasperini, M. Giovannini, V. F. Mukhanov, and G. Veneziano, Phys. Rev. D 51, 6744 (1995).

[51] C.-P. Ma and E. Bertschinger, Astrophys. J. 455, 7 (1995).

[52] A. Lukas, B. A. Ovrut, and D. Waldram, Nucl. Phys. B532, 43 (1998).

[53] A. Lukas, B. A. Ovrut, and D. Waldram, Nucl. Phys. B540, 230 (1999).

[54] S. Mukohyama, Phys. Rev. D 62, 084015 (2000).

[55] H. Kodama, A. Ishibashi, and O. Seto, Phys. Rev. D 62, 064022 (2000).

[56] D. Langlois, Phys. Rev. D 62, 126012 (2000).

[57] R. Maartens, Phys. Rev. D 62, 084023 (2000). 